Homology, Homotopy and Applications, vol.14(1), 2012, pp.263-280

\title{
CLASS-COMBINATORIAL MODEL CATEGORIES
}

\author{
BORIS CHORNY AND JIŘÍ ROSICKÝ
}

\author{
(communicated by J. Daniel Christensen)
}

\begin{abstract}
We develop an extension of the framework of combinatorial model categories. The category of small presheaves over large indexing categories and ind-categories are the basic examples of non-combinatorial model categories embraced by the new machinery called class-combinatorial model categories.

The definition of the new class of model categories is based on the corresponding extension of the theory of locally presentable and accessible categories developed in the companion paper, where we introduced the concepts of class-locally presentable and class-accessible categories.

In this work we prove that the category of weak equivalences of a nice class-combinatorial model category is class-accessible. Our extension of J. Smith's localization theorem depends on the verification of a cosolution-set condition. The deepest result is that the (left Bousfield) localization of a class-combinatorial model category with respect to a strongly class-accessible localization functor is class-combinatorial again.
\end{abstract}

\section{Introduction}

The theory of combinatorial model categories pioneered by J. Smith in the end of 1990s has become a standard framework for abstract homotopy theory. The foundations of the subject may be found in $[\mathbf{2}]$ and $[\mathbf{1 4}, \mathbf{1 5}]$; a concise exposition has appeared in $[\mathbf{2 5}, \mathrm{A} .2 .6]$.

A model category $\mathcal{M}$ is combinatorial if it satisfies two conditions. The first condition requires that the underlying category $\mathcal{M}$ be locally presentable (see, e.g., $[\mathbf{1}]$ for the definition and an introduction to the subject). The second condition demands that the model structure be cofibrantly generated (see, e.g., $[\mathbf{2 0}]$ for the definition and discussion).

Several interesting examples of non-combinatorial model categories without Quillen equivalent combinatorial models appeared over the past decade. For example the categories of pro-spaces and ind-spaces were applied in new contexts in homotopy theory $[\mathbf{1 2}, \mathbf{2 1}]$ resulting in non-cofibrantly generated model structures constructed on non-locally presentable categories. The maturation of the calculus of homotopy

Received October 25, 2011, revised March 1, 2012; published on June 7, 2012. 2000 Mathematics Subject Classification: 18G55, 55P60.

Key words and phrases: class-cofibrantly generated, model category, localization.

Article available at http://intlpress.com/HHA/v14/n1/a13 and doi:10.4310/HHA.2012.v14.n1.a13

Copyright (C) 2012, International Press. Permission to copy for private use granted. 
functors $[\mathbf{1 7}]$ stimulated the development of the abstract homotopy theory of small functors over large categories [10] resulting in formulation of the basic ideas of Goodwillie calculus in the language of model categories [3]. The model categories used for this purpose are also not cofibrantly generated and the underlying category of small functors from spaces to spaces (or spectra) is not a locally presentable category.

However, all the model categories from the examples above are class-cofibrantly generated (except for the pro-categories, which are class-fibrantly generated). This extension of the classical definition was introduced in [8], which in turn developed the ideas by E. Dror Farjoun originated in the equivariant homotopy theory [16].

The purpose of the current paper is to develop a framework extending J. Smith's combinatorial model categories, so that the model categories of small presheaves on large categories and ind-categories of model categories (the opposite categories of pro-categories) would become the examples of the newly defined class-combinatorial model categories. The definition of a class-combinatorial model category consists, similarly to that of a combinatorial model category, of two conditions: the underlying category is required to be class-locally presentable and the model structure must be class-cofibrantly generated. As we mentioned above, the second condition was studied in the earlier work $[\mathbf{8}]$, while the first condition relies on a concept of class-locally presentable category. This was introduced and studied in the companion project [11], which is a prerequisite for reading this paper. Informally, a class-accessible category is a category closed under sufficiently large filtered colimits and equipped with a class of generating objects with uniformly bounded presentability ranks, such that every object in the category is a sufficiently large filtered colimit of generating objects. A class-accessible category is called class-locally presentable if in addition we require existence of all limits and colimits. See [11, Def. 2.1] for the formal introduction and discussion of these concepts.

The main results of our paper generalize the corresponding results about the combinatorial model categories. First, in Theorem 2.12 we prove that the levelwise weak equivalences in the category of small presheaves form a class-accessible category (see [11] for the definition). In Remark 2.14 we formulate mild conditions which guarantee that a class-combinatorial model category has a class-accessible subcategory of weak equivalences. Such class-combinatorial model categories are called nice in this paper. Then we prove a class-version of Smith's theorem on existence of combinatorial model structures $[\mathbf{2}, 1.7]$ in Theorem 2.15 .

The central result of J. Smith's theory is the localization theorem, stating the existence of the (left Bousfield) localization of any combinatorial model category with respect to any set of maps. After a brief discussion of construction of localization functors with respect to cone-coreflective classes of cofibrations with bounded presentability ranks of domains and codomains, we prove in Theorem 3.10 a variant of a localization theorem for nice class-combinatorial model categories with respect to strongly class-accessible homotopy localization functors (i.e., localization functors preserving $\lambda$-filtered colimits and $\lambda$-presentable objects for some cardinal $\lambda$ ). Although an application of our localization theorem depends on the verification of a cosolution-set condition for the class of intended generating trivial cofibrations, we are able to check this condition in many interesting situations. In the last Theorem 3.13 we prove that in the cases where the localization with respect to a strongly class-accessible functor exists, the localized model category is class-combinatorial again. 
We conclude the paper by several examples of localized model categories. Using Theorem 3.13 we show that the $n$-polynomial model category constructed in $[\mathbf{3}]$ is class-combinatorial (Example 3.15). On the other hand, there is a model category constructed in [9] as a localization of a class-combinatorial model category with respect to an inaccessible localization functor that happens to be non-class-cofibrantly generated (Example 3.16).

\section{Class-combinatorial model categories}

Recall that the notion of a weak factorization system being class-cofibrantly generated was introduced in [11, Def. 4.7]. We repeat this definition here, since it is of central importance for this section.

Definition 2.1. A weak factorization system $(\mathcal{L}, \mathcal{R})$ in a class-locally $\lambda$-presentable category $\mathcal{K}$ was called class-cofibrantly $\lambda$-generated if $\mathcal{L}=\operatorname{cof}(\mathcal{C})$ for a cone-coreflective class $\mathcal{C}$ of morphisms such that

(1) morphisms from $\mathcal{C}$ have $\lambda$-presentable domains and codomains and

(2) any morphism between $\lambda$-presentable objects has a weak factorization with the middle object $\lambda$-presentable.

To be cone-coreflective means for each $f$ there is a subset $\mathcal{C}_{f}$ of $\mathcal{C}$ such that each morphism $g \rightarrow f$ in $\mathcal{K} \rightarrow$ with $g \in \mathcal{C}$ factorizes as

$$
g \rightarrow h \rightarrow f
$$

with $h \in \mathcal{C}_{f}$.

If the weak factorization is functorial, a cofibrantly class- $\lambda$-generated weak factorization system is cofibrantly class- $\mu$-generated for each regular cardinal $\mu \unrhd \lambda$. Without functoriality, condition (2) does not need to go up to $\mu$ and thus we will make it a part of the following definition.

Definition 2.2. Let $\mathcal{K}$ be a class locally- $\lambda$-presentable model category. We say that $\mathcal{K}$ is class- $\lambda$-combinatorial if both (cofibrations, trivial fibrations) and (trivial cofibrations, fibrations) are cofibrantly class- $\mu$-generated weak factorization systems for every regular cardinal $\mu \unrhd \lambda$. If in addition $\mathcal{K}$ is a simplicial category, then we understand the completeness and cocompleteness assumptions in the enriched sense, in particular, $\mathcal{K}$ is tensored and cotensored over the simplicial sets.

A model category is called class-combinatorial if it is class- $\lambda$-combinatorial for some regular cardinal $\lambda$.

Any combinatorial model category is class-combinatorial. The reason is that weak factorizations are functorial and, moreover, the resulting functors are accessible. Thus they are strongly accessible and this property goes up for $\lambda \triangleleft \mu$ (cf. [27]).

Example 2.3. Let SSet denote the category of simplicial sets. Given a simplicial category $\mathcal{A}$, we denote by $\mathcal{P}(\mathcal{A})$ the category of small simplicial presheaves on $\mathcal{A}$. The objects are functors $\mathcal{A}^{\mathrm{op}} \rightarrow$ SSet which are small weighted colimits of simplicial representable functors. Although in [11] we used this notation for small set-valued presheaves, in this paper we reserve the notation $\mathcal{P}(\mathcal{A})$ only for small simplicial 
presheaves in order to avoid confusion. In the rare occasions when we need to use the category of small set-valued presheaves on $\mathcal{A}$, we denote it by $\mathcal{P}_{0}(\mathcal{A})$.

The simplicial category $\mathcal{P}(\mathcal{A})$ is complete provided that $\mathcal{A}$ is complete (see [13]); completeness is meant in the enriched sense (see, e.g., [4] or $[\mathbf{2 3}]$ ).

The category $\mathcal{P}(\mathcal{A})$ is always class-finitely-accessible, because each small simplicial presheaf is a conical colimit of presheaves from $\mathcal{G}=\left\{\operatorname{hom}_{\mathcal{A}}(-, A) \otimes K \mid A \in \mathcal{A}\right.$, $K \in \mathbf{S S e t}\}$. Therefore each small presheaf is a filtered colimit of finite colimits of elements of $\mathcal{G}$. The elements of $\mathcal{G}$ are, in turn, filtered colimits of the elements of $\left.\mathcal{G}_{\text {fin }}=\left\{\operatorname{hom}(-, A) \otimes L \mid A \in \mathcal{A}, L \in \mathbf{S S e t}_{\text {fin }}\right)\right\}$, where $\mathbf{S S e t}_{\text {fin }}$ denotes the full subcategory of SSet consisting of finitely presentable simplicial sets. Therefore, every small presheaf is a filtered colimit of finite colimits of the elements of $\mathcal{G}_{\text {fin }}$.

We are going to show that, for a complete simplicial category $\mathcal{A}$, the category $\mathcal{P}(\mathcal{A})$ equipped with the projective model structure is class-combinatorial. We will need the following result.

Lemma 2.4. Let $\mathcal{K}$ be a class locally $\lambda$-presentable simplicial category. Then $\mu$ presentable objects in $\mathcal{K}$ are closed under finite conical limits for every uncountable regular cardinal $\mu \unrhd \lambda$. In the case that $\mathcal{K}=\mathcal{P}(\mathcal{A})$ the $\mu$-presentable functors are closed under finite weighted limits.

Proof. Finite conical limits in $\mathcal{K}$ coincide with finite limits in the underlying category $\mathcal{K}_{0}$, since we assume that $\mathcal{K}$ is tensored over the simplicial sets as part of the completeness assumption [23, 3.8]. We intend to use a similar statement proved earlier in presheaf categories. For this purpose we apply the limit preserving embedding constructed in $[\mathbf{1 1}, 2.6] E: \mathcal{K}_{0} \rightarrow \mathcal{P}_{0}(\mathcal{A})$, where $\mathcal{A}=\operatorname{pres}_{\lambda} \mathcal{K}_{0}$.

Let $F: \mathcal{D} \rightarrow \mathcal{K}_{0}$ be a finite diagram of $\mu$-presentable objects. If we denote $P=$ $\lim _{D \in \mathcal{D}} F(D)$, then $E P=\lim _{D \in \mathcal{D}} E F(D)$, where $E F$ is a diagram of $\mu$-presentable objects, since $E$ is strongly $\lambda$-accessible, hence strongly $\mu$-accessible by $[\mathbf{1 1}, 2.8]$, because $\mu \unrhd \lambda$.

Consider the category $\mu$-colex $(\mathcal{A})$, which is a free completion of $\mathcal{A}$ under $\mu$-small colimits, as a full subcategory of $\mu$-presentable objects of the free completion of $\mathcal{A}$ under colimits $\mathcal{P}_{0}(\mathcal{A})$ (by a simple observation that every $\mu$-small colimit of representables is $\mu$-small and a more technical converse statement proved in [26, pp. 35-37]. Observe that every limit existing in $\mu$-colex $(\mathcal{A})$ remains a limit in $\mathcal{P}_{0}(\mathcal{A})$, in other words, the full embedding constructed above preserves limits. We argue as follows. If $L$ is an inverse limit of a diagram in $\mu$-colex $(\mathcal{A})$ and $L^{\prime}$ is a possible candidate for an inverse limit of the same diagram in $\mathcal{P}_{0}(\mathcal{A})$, then $L^{\prime}$ may be represented as a $\mu$-filtered colimit of $\mu$-presentable presheaves, i.e., elements of $\mu$-colex $(\mathcal{A}): L^{\prime}=\operatorname{colim}_{i} P_{i}$. The natural map $L \rightarrow L^{\prime}$ has an inverse, since everyone of $P_{i}$ 's admits a natural map into $L$, because it forms a cone over the original diagram through the map $P_{i} \rightarrow L^{\prime}$, and together these maps define a natural map $L^{\prime} \rightarrow L$. We conclude that $L \cong L^{\prime}$ by naturality considerations.

The category $\mu$-colex $(\mathcal{A})$ is closed under finite limits by $[\mathbf{2 2}, 4.9]$, hence $\mu$-presentable objects in $\mathcal{P}_{0}(\mathcal{A})$ are closed under finite limits. In other words we have shown that $E P$ is $\mu$-presentable, but we need to show that $P$ is $\mu$-presentable. Let $K=\underset{i}{\operatorname{colim}} K_{i}$ 
be a $\mu$-filtered colimit, then

$$
\begin{aligned}
\operatorname{hom}(P, K)= & \operatorname{hom}(E P, E K)=\operatorname{hom}\left(E P, \operatorname{colim}_{i} E\left(K_{i}\right)\right)= \\
& \operatorname{colim}_{i} \operatorname{hom}\left(E P, E K_{i}\right)=\operatorname{colim}_{i} \operatorname{hom}\left(P, K_{i}\right) .
\end{aligned}
$$

Now we turn to the case $\mathcal{K}=\mathcal{P}(\mathcal{A})$. A weight $W: \mathcal{D} \rightarrow$ SSet is finite if $\mathcal{D}$ has finitely many objects and all its hom-objects $\mathcal{D}(c, d)$ and all values $W(d)$ are finitely presentable simplicial sets (see $[\mathbf{2 4}, 4.1]$ ) Following [24, 4.3], finite weighted limits can be constructed from finite conical limits and cotensors with finitely presentable simplicial sets. Thus we have to show that $\mu$-presentable objects in $\mathcal{K}$ are closed under these limits.

It remains to show that the $\mu$-presentable functors are closed under cotensors with finitely presentable simplicial sets. The later are finite conical colimits of $\Delta_{n}$, $n=0,1,2, \ldots$. Thus we have to show that $\mu$-presentable objects in $\mathcal{P}(\mathcal{A})$ are closed under cotensors with $\Delta_{n}$ 's.

Let $H$ be $\mu$-presentable in $\mathcal{P}(\mathcal{A})$. Since $H$ is a $\mu$-small colimit of tensors $H_{i}$ of finitely presentable simplicial sets with representables and both colimits and cotensors in $\mathcal{P}(\mathcal{A})$ are pointwise, we have

$$
\begin{aligned}
H^{\Delta_{n}}(A) & =\left(\operatorname{colim} H_{i}\right)^{\Delta_{n}}(A)=\left(\operatorname{colim} H_{i}(A)\right)^{\Delta_{n}} \\
& =\operatorname{hom}\left(\Delta_{n}, \operatorname{colim} H_{i}(A)\right) \cong \operatorname{colim} \operatorname{hom}\left(\Delta_{n}, H_{i}(A)\right) \\
& =\operatorname{colim} H_{i}(A)^{\Delta_{n}}=\left(\operatorname{colim} H_{i}^{\Delta_{n}}\right)(A)
\end{aligned}
$$

for each $A$ in $\mathcal{A}$. Hence

$$
H^{\Delta_{n}} \cong \operatorname{colim} H_{i}^{\Delta_{n}}
$$

and thus it suffices to show that each $H_{i}^{\Delta_{n}}$ is $\mu$-presentable. Since each $H_{i}$ is equal to $V \otimes \operatorname{hom}(-, B)$ for some finitely presentable simplical set $V$ and $B$ in $\mathcal{A}$, we get for the same reasons as above

$$
\begin{aligned}
& \operatorname{hom}\left(\Delta_{n}, V \otimes \operatorname{hom}(A, B)\right)=\operatorname{hom}\left(\Delta_{n}, V \times \operatorname{hom}(A, B)\right) \cong \\
& \operatorname{hom}\left(\Delta_{n}, V\right) \times \operatorname{hom}\left(\Delta_{n}, \operatorname{hom}(A, B)\right) \cong V^{\Delta_{n}} \times \operatorname{hom}\left(A, B^{\Delta_{n}}\right)= \\
& \left(V^{\Delta_{n}} \otimes \operatorname{hom}\left(-, B^{\Delta_{n}}\right)\right)(A)
\end{aligned}
$$

and thus

$$
(V \otimes \operatorname{hom}(-, B))^{\Delta_{n}} \cong V^{\Delta_{n}} \otimes \operatorname{hom}\left(-, B^{\Delta_{n}}\right) .
$$

The latter objects are $\mu$-presentable.

Remark 2.5. It is possible to use the same argument as in the proof above for cotensors with finitely presentable simplicial sets in an arbitrary class-locally presentable simplicial category $\mathcal{K}$, which is cotensored over the simplicial sets as we assume that $\mathcal{K}$ is cocomplete in the enriched sense. In order to implement it we need to show that the class of $\lambda$-presentable objects in $\mathcal{K}$ is dense, so that there would exist an embedding $\mathcal{K} \rightarrow \mathcal{P}(\mathcal{A})$ preserving $\lambda$-filtered colimits. The density of the subcategory of $\lambda$-presentable objects of $\mathcal{K}$ in the enriched settings may be verified similarly to the analogous treatment of locally presentable categories $[\mathbf{2 4}, 7.2,7.3]$. 
Proposition 2.6. Let $\mathcal{A}$ be a complete simplicial category. Then $\mathcal{P}(\mathcal{A})$ is class- $\lambda$ combinatorial with respect to the projective model structure for each uncountable regular cardinal $\lambda$.

Proof. Following $[\mathbf{1 0}], \mathcal{P}(\mathcal{A})$ is a model category where the generating classes $\mathcal{I}$ and $\mathcal{J}$ of cofibrations and trivial cofibrations are cone-coreflective and satisfy condition 2.1(1) for any regular cardinal $\lambda$. In fact, $\mathcal{I}$ consists of morphisms

$$
\partial \Delta_{n} \otimes \operatorname{hom}(-, A) \rightarrow \Delta_{n} \otimes \operatorname{hom}(-, A)
$$

and $\mathcal{J}$ of morphisms

$$
\Lambda_{n}^{k} \otimes \operatorname{hom}(-, A) \rightarrow \Delta_{n} \otimes \operatorname{hom}(-, A),
$$

and all involved domains and codomains are finitely presentable. We have to show that they satisfy 2.1(2) as well, i.e., that they are bounded. Let $\lambda$ be uncountable and $f: G \rightarrow H$ be a morphism between $\lambda$-presentable objects and consider a morphism $g \rightarrow f$, where $g \in \mathcal{I}$. Following the proof of 3.7 in [10], this morphism corresponds to a morphism $\operatorname{hom}(-, A) \rightarrow P$, where $P$ is the pullback

$$
G^{\partial \Delta_{n}} \times_{H^{\partial \Delta_{n}}} H^{\Delta_{n}}
$$

Since $P$ is $\lambda$-presentable (see 2.4), there is a choice of a set $\mathcal{T}_{f}$ from [11] $4.8(2)$ whose cardinality does not exceed $\lambda$. Since all morphisms from $\mathcal{I}$ have finitely presentable domains and codomains, the factorization of $f$ stops at $\omega$. Thus the cardinality of $\mathcal{T}_{f}^{*}$ is smaller than $\lambda$. Following [11] 4.8 (2), condition [11] $3.7(2)$ is satisfied. The argument for $\mathcal{J}$ is the same.

Remark 2.7. A very useful property of a combinatorial model categories is that the class of weak equivalences is an accessible and accessibly embedded subcategory of the category of morphisms $\mathcal{K} \rightarrow$ (see $[\mathbf{2 8}, 4.1]$ or $[\mathbf{2 5}$, A2.6.6]). Together with Smith's theorem [2] this implies the localization theorem for combinatorial model categories with respect to sets of maps. It would be natural to expect that a similar property holds in the class-combinatorial situation. Unfortunately we were unable to prove it in this generality. But in many interesting situations we are able to prove that the class of weak equivalences is a class-accessible subcategory of the category of morphisms.

Lemma 2.8. Let $\mathcal{A}$ be a complete simplicial category. Then $\mathcal{P}(\mathcal{A})$ admits a strongly class-accessible fibrant replacement functor.

Proof. The functor $\mathrm{Ex}^{\infty}$ : SSet $\rightarrow$ SSet is a finitely accessible fibrant replacement simplicial functor (see [18]). For a small simplicial functor $F: \mathcal{A}^{\text {op }} \rightarrow$ SSet, let $\operatorname{Fib}(F)$ be the composition

$$
\mathcal{A}^{\mathrm{op}} \stackrel{F}{\longrightarrow} \text { SSet } \stackrel{E x^{\infty}}{\longrightarrow} \text { SSet . }
$$

We will show that this composition is small.

The category of finitely accessible simplicial functors SSet $\rightarrow$ SSet is equiva-

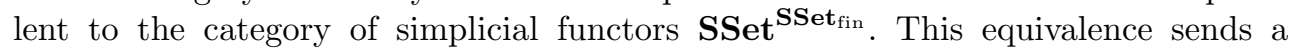
finitely accessible functor SSet $\rightarrow$ SSet to its restriction to $\mathbf{S S e t}_{\text {fin }}$. Thus homfunctors $\operatorname{hom}(S,-)$ : SSet $\rightarrow$ SSet with $S$ finitely presentable correspond to homfunctors $\operatorname{hom}(S,-): \mathbf{S S e t}_{\text {fin }} \rightarrow \mathbf{S S e t}$. Since every simplical functor $\mathbf{S S e t}_{\text {fin }} \rightarrow$ SSet 
is a weighted colimit of hom-functors, every finitely accessible simplicial functor SSet $\rightarrow$ SSet is a weighted colimit of hom-functors $\operatorname{hom}(S,-)$ with $S$ finitely presentable. Thus the composition $\operatorname{Ex}^{\infty} F$ is a weighted colimit of functors $\operatorname{hom}(S,-) F$ with $S$ finitely presentable. But the functor $\operatorname{hom}(S,-) F$ is small because it is isomorphic to the cotensor $F^{S}$. The reason is that natural transformations

$$
\mathcal{A}(-, A) \rightarrow \operatorname{hom}(S,-) F=\operatorname{hom}(S, F-)
$$

correspond to morphisms $S \rightarrow F A$, i.e., to morphisms

$$
S \rightarrow \mathcal{P}(\mathcal{A})(\mathcal{A}(-, A), F)
$$

which, by the definition of the cotensor, correspond to morphisms $\mathcal{A}(-, A) \rightarrow F^{S}$. Consequently, $\mathrm{Ex}^{\infty} F$ is small as a weighted colimit of small functors.

We have obtained the functor Fib: $\mathcal{P}(\mathcal{A}) \rightarrow \mathcal{P}(\mathcal{A})$ which clearly has fibrant values. Moreover, the pointwise trivial cofibration

$$
\mathrm{Id}_{\text {SSet }} \rightarrow \mathrm{Ex}^{\infty}
$$

yields a weak equivalence $\operatorname{Id}_{\mathcal{P}(\mathcal{A})} \rightarrow$ Fib. Thus Fib is a fibrant replacement functor on $\mathcal{P}(\mathcal{A})$. Since $\mathrm{Ex}^{\infty}$ is finitely accessible, so is Fib. We know that $\mathrm{Ex}^{\infty}$ is a weighted colimit of hom-functors $\operatorname{hom}(S,-)$ with $S$ finitely presentable. The corresponding weight is $\lambda$-small for an uncountable regular cardinal $\lambda$. Let $F$ be $\lambda$-presentable in $\mathcal{P}(\mathcal{A})$. Then $\operatorname{Fib}(F)$ is a $\lambda$-small weighted colimit of $\operatorname{hom}(S,-) F \cong F^{S}$ and the latter functors are $\lambda$-presentable following 2.4. Hence $\operatorname{Fib}(F)$ is $\lambda$-presentable (the argument is analogous to $[\mathbf{2 4}], 4.14)$. Thus Fib is strongly class- $\lambda$-accessible.

Definition 2.9. Let $\mathcal{A}$ be a complete simplicial category and $f: A \rightarrow B$ be a morphism in $\mathcal{P}(\mathcal{A})$. The mapping cocylinder on $f$ is the object $S(f)$ of $\mathcal{P}(\mathcal{A})$ defined as a pullback

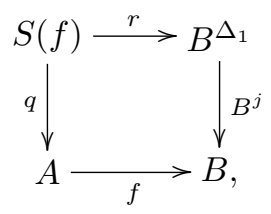

where $j: \Delta_{0} \rightarrow \Delta_{1}$ sends 0 to 0 .

Remark 2.10. Mapping cocylinders were used in the $\mathrm{PhD}$ thesis of J.P. Serre in order to replace an arbitrary map of topological spaces by a fibration. We are going to use them basically for the same purpose in $\mathcal{P}(\mathcal{A})$. The advantage over the modern methods of factorization is the functoriality of $S(-)$.

Lemma 2.11. Let $\mathcal{A}$ be a complete simplicial category and $f: A \rightarrow B$ a morphism of fibrant objects in $\mathcal{P}(\mathcal{A})$. Then there exists a factorization

$$
A \stackrel{i}{\longrightarrow} S(f) \stackrel{p}{\longrightarrow} B
$$

of $f$, where $i$ is a weak equivalence and $p$ is a fibration. 
Proof. The pullback in 2.9 may be split in two pulbacks

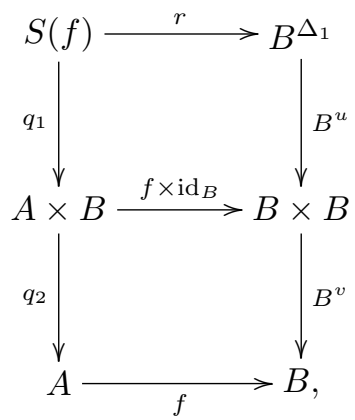

where

$$
\Delta_{0} \stackrel{v}{\longrightarrow} \Delta_{0}+\Delta_{0} \stackrel{u}{\longrightarrow} \Delta_{1}
$$

is the factorization of $j$. Since both $u$ and $v$ are cofibrations and $B$ is fibrant, the vertical morphisms $B^{u}$ and $B^{v}$ are fibrations (see [19] 9.3 .9 (2a)). Moreover, since $j$ is a trivial cofibration, $B^{j}$ is a trivial fibration. Thus $q_{1}$ and $q_{2}$ are fibrations and $q=q_{2} g_{1}$ is a trivial fibration.

Let $t$ denote the unique morphism $\Delta_{1} \rightarrow \Delta_{0}$. Since,

$$
B^{j} f^{\Delta_{1}} A^{t}=f A^{j} A^{t}=f,
$$

there is a unique morphism $i: A \rightarrow S(f)$ such that $q i=\operatorname{id}_{A}$ and $r i=f^{\Delta_{1}} A^{t}$. Since $q$ is a trivial fibration, $i$ is a weak equivalence. Since $B^{v}: B \times B \rightarrow B$ is the first projection of the product, $q_{2}: A \times B \rightarrow A$ is the first projection as well. Let $p_{2}: A \times$ $B \rightarrow B, \bar{p}_{2}: B \times B \rightarrow B$ be the second projections and $v^{\prime}: \Delta_{0} \rightarrow \Delta_{0}+\Delta_{0}$ be the second injection of the coproduct. Then $\bar{p}_{2}=B^{v^{\prime}}$ and

$$
\begin{aligned}
p_{2} q_{1} i & =\bar{p}_{2}\left(f \times \operatorname{id}_{B}\right) q_{1} i=\bar{p}_{2} B^{u} r i=\bar{p}_{2} B^{u} f^{\Delta_{1}} A^{t}=B^{v^{\prime}} B^{u} f^{\Delta_{1}} A^{t} \\
& =B^{u v^{\prime}} f^{\Delta_{1}} A^{t}=f A^{u v^{\prime}} A^{t}=f .
\end{aligned}
$$

Since $B$ is fibrant, $p_{2}$ is a fibration and thus $p=p_{2} q_{1}$ is a fibration. We have $f=p i$.

Theorem 2.12. Let $\mathcal{A}$ be a complete simplicial category and denote by $\mathcal{W}$ the class of weak equivalences in the projective model structure on $\mathcal{P}(\mathcal{A})$. Then $\mathcal{W}$ is a classaccessible category strongly accessibly embedded in $\mathcal{P}(\mathcal{A}) \rightarrow$.

Proof. Let Fib: $\mathcal{P}(\mathcal{A}) \rightarrow \mathcal{P}(\mathcal{A})$ be the strongly class-accessible fibrant replacement functor constructed in 2.8. Consider the functor

$$
R: \mathcal{P}(\mathcal{A}) \rightarrow \mathcal{P}(\mathcal{A}) \rightarrow
$$

assigning to a morphism $f: A \rightarrow B$ the fibration $p: S(\operatorname{Fib}(f)) \rightarrow \operatorname{Fib}(B)$ from 2.11. Since the construction of $S(f)$ uses only finite limits, the functor $S(-): \mathcal{P}(\mathcal{A}) \rightarrow \rightarrow$ $\mathcal{P}(\mathcal{A})$ is strongly class-accessible by 2.4 . Therefore the functor $R(-)$ is also strongly class-accessible. A morphism $\alpha: F \rightarrow G$ in $\mathcal{P}(\mathcal{A})$ is a weak equivalence if and only if $\operatorname{Fib}(f)$ is a weak equivalence, hence if and only if $R(f)$ is a trivial fibration. 
Let $\mathcal{F}_{0}$ denote the full subcategory of $\mathcal{P}(\mathcal{A}) \rightarrow$ consisting of trivial fibrations. Following 2.6 and [11] $4.9, \mathcal{F}_{0}$ is class- $\lambda$-accessible and strongly $\lambda$-accessibly embedded in $\mathcal{P}(\mathcal{A}) \rightarrow$ for every uncountable regular cardinal $\lambda$. Since $\mathcal{W}$ is given by the pullback

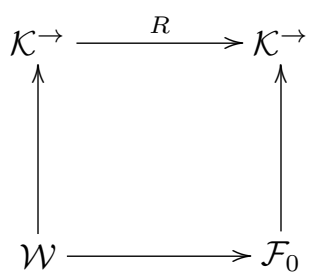

whose vertical leg on the right is transportable, $\mathcal{W}$ is equivalent to the pseudopullback (see [11] 3.2). Thus, [11] 3.1 implies that $\mathcal{W}$ is a class-accessible subcategory of $\mathcal{P}(\mathcal{A}) \rightarrow$.

Definition 2.13. A class-combinatorial model category $\mathcal{K}$ is nice if the class of weak equivalences $\mathcal{W}$ is a class-accessible, strongly accessibly embedded subcategory of $\mathcal{K} \rightarrow$.

Remark 2.14. We have just proved that $\mathcal{P}(\mathcal{A})$ equipped with the projective model structure is a nice model category for any complete simplicial category $\mathcal{A}$. The same argument applies to every simplicial class-combinatorial model category which is equipped with a strongly class-accessible fibrant replacement functor and whose $\mu$ presentable objects are closed under finite weighted limits for each $\mu \unrhd \lambda$ (where $\lambda$ is a cardinal). We are not aware of any example of a class-combinatorial model category, which fails to be nice.

We now prove a version of Smith's theorem $[\mathbf{2}, 1.7]$ in the class-combinatorial case.

Theorem 2.15. Let $\mathcal{K}$ be a class-locally $\lambda$-presentable category, $\mathcal{I}$ a $\lambda$-bounded class of morphisms and $\mathcal{W}$ a class of morphisms of $\mathcal{K}$ such that

(1) $\mathcal{W}$ is a class- $\lambda$-accessible and strongly $\lambda$-accessibly embedded subcategory of $\mathcal{K} \rightarrow$ with the 2-out-of-3 property,

(2) $\mathcal{I}^{\square} \subseteq \mathcal{W}$, and

(3) $\operatorname{cof}(\mathcal{I}) \cap \mathcal{W}$ is closed under pushout and transfinite composition and cone-coreflective in $\mathcal{K} \rightarrow$.

Then, taking $\operatorname{cof}(\mathcal{I})$ for cofibrations and $\mathcal{W}$ for weak equivalences, we get a model category structure on $\mathcal{K}$.

Proof. Since $\mathcal{I}$ is $\lambda$-bounded, $\left(\operatorname{cof}(\mathcal{I}), \mathcal{I}^{\square}\right)$ is a cofibrantly class- $\lambda$-generated weak factorization system. For every $\lambda$-presentable $w \in \mathcal{W}$, we construct a factorization in $\mathcal{K}$ into a cofibration $j$ followed by a trivial fibration. By 2-out-of-3 property for $\mathcal{W}$ and (2), $j$ is in $\mathcal{W}$. Let $\mathcal{J}$ be the class of these morphisms $j$ for all $\lambda$-presentable $w \in \mathcal{W}$.

We will check now the conditions of Lemma $[\mathbf{2}, 1.8]$. We have to show that for every morphism $i \rightarrow w$ in $\mathcal{K} \rightarrow$ with $i \in \mathcal{I}$ and $w \in \mathcal{W}$ there exists $j \in \mathcal{J}$ that factors it $i \rightarrow j \rightarrow w$. First note that there exists a $\lambda$-presentable $w^{\prime} \in \mathcal{W}$, which factors the original morphism, since every $w$ is a $\lambda$-filtered colimit of $\lambda$-presentable objects $\mathcal{W}$ 
and every $i \in I$ is $\lambda$-presentable in $\mathcal{K} \rightarrow$; we used here that the inclusion of $\mathcal{W}$ to $\mathcal{K} \rightarrow$ preserves $\lambda$-presentable objects. Next, decompose that morphism $w^{\prime}$ into a cofibration $j \in \mathcal{J}$ followed by a trivial fibration. The lifting axiom in $\mathcal{K}$ finishes this argument.

Lemma $[2,1.8]$ implies that $\operatorname{cof} \mathcal{J}=\operatorname{cof} \mathcal{I} \cap \mathcal{W}$. The requirement that $\operatorname{cof} \mathcal{I} \cap \mathcal{W}$ is cone-coreflective in $\mathcal{K} \rightarrow$ ensures that $\mathcal{J}$ is cone-coreflective as well (by the same argument as above). By construction, the domains of all the elements in $\mathcal{J}$ are $\lambda$ presentable. Hence $\mathcal{J}$ satisfies the assumptions of [11] 4.3 and thus $\left(\operatorname{cof}(\mathcal{J}), \mathcal{J}^{\square}\right)$ is a weak factorization system. Since $\mathcal{W}$ is closed under retracts in $\mathcal{K} \rightarrow$ (cf. [1] 2.4 and 2.5 ), we get a model category structure on $\mathcal{K}$.

Remark 2.16. Let $\mathcal{K}$ be a locally presentable category, $\mathcal{I}$ a set of morphisms and $\mathcal{W}$ a class of morphisms of $\mathcal{K}$ such that

(1) $\mathcal{W}$ has the 2-out-of-3 property and is closed under retracts in $\mathcal{K} \rightarrow$,

(2) $\mathcal{I}^{\square} \subseteq \mathcal{W}$, and

(3) $\operatorname{cof}(\mathcal{I}) \cap \mathcal{W}$ is closed under pushout and transfinite composition.

Then, taking $\operatorname{cof}(\mathcal{I})$ for cofibrations and $\mathcal{W}$ for weak equivalences, we get a combinatorial model category if and only if the inclusion of $\mathcal{W}$ in $\mathcal{K} \rightarrow$ is accessible. This is the content of Smith's theorem (see [2] for sufficiency and $[\mathbf{2 5}]$ or $[\mathbf{2 8}]$ for necessity).

We do not know whether this can be generalized to class-accessible setting and 2.15 is what we are able to do. The question is whether cone-coreflectivity of $\operatorname{cof}(\mathcal{I}) \cap \mathcal{W}$ follows from the other assumptions. We also do not know whether the model category in 2.15 is class-combinatorial. Indeed, we only know that the class $\mathcal{J}$ satisfies 2.1(1).

\section{Left Bousfield localizations}

Recall that $\tilde{h}$ is a cofibrant approximation of $h$ if there is a commutative square

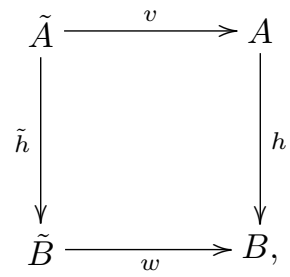

where $v$ and $w$ are weak equivalences and $\tilde{A}, \tilde{B}$ are cofibrant.

Definition 3.1. Let $\mathcal{K}$ be a class-combinatorial simplicial model category and $\mathcal{F}$ a class of morphisms of $\mathcal{K}$. Assume that $\mathcal{F}$ contains only cofibrations between cofibrant objects. An object $K$ in $\mathcal{K}$ is called $\mathcal{F}$-local if it is fibrant and

$$
\operatorname{hom}(f, K): \operatorname{hom}(B, K) \rightarrow \operatorname{hom}(A, K)
$$

is a weak equivalence of simplicial sets for each $f: A \rightarrow B$ in $\mathcal{F}$.

A morphism $h$ of $\mathcal{K}$ is called an $\mathcal{F}$-local equivalence if $\operatorname{hom}(\tilde{h}, K)$ is a weak equivalence for each $\mathcal{F}$-local object $K$; here, $\tilde{h}$ is a cofibrant approximation of $h$.

The full subcategory of $\mathcal{K}$ consisting of $\mathcal{F}$-local objects is denoted $\operatorname{Loc}(\mathcal{F})$ and the full subcategory of $\mathcal{K} \rightarrow$ consisting of $\mathcal{F}$-local equivalences is denoted $\operatorname{LEq}(\mathcal{F})$. 
We say that there exists a left Bousfield localization of $\mathcal{F}$ if cofibrations in $\mathcal{K}$ and $\mathcal{F}$-local equivalences form a model category structure on $\mathcal{K}$.

Remark 3.2. (1) It is easy to see that the definition of a local $\mathcal{F}$-equivalence does not depend on the choice of a cofibrant approximation.

(2) Following [19, 9.3.3(2)], any weak equivalence in $\mathcal{K}$ is an $\mathcal{F}$-local equivalence. On the other hand, every $\mathcal{F}$-local equivalence between $\mathcal{F}$-local objects is a weak equivalence in $\mathcal{K}$ (cf. [19,3.2.13]).

(3) If $\mathcal{K}$ is left proper then the intersection of cofibrations and $\mathcal{F}$-local equivalences is closed under pushout and transfinite composition (see [19], 13.3.10, 17.9.4 for a non-trivial part of the proof); the trivial part is that hom $(-, K)$ sends colimits to limits and cofibrations to fibrations. It is also closed under retracts in $\mathcal{K} \rightarrow$ of course.

Given a morphism $f,\{f\}$-local objects are called $f$-local and analogously for $f$ local equivalences. The corresponding categories are called $\operatorname{Loc}(f)$ and $\operatorname{LEq}(f)$.

Proposition 3.3. Let $\mathcal{K}$ be a class-combinatorial simplicial model category and $\mathcal{F}$ a set of cofibrations between cofibrant objects of $\mathcal{K}$. Then $\operatorname{Loc}(\mathcal{F})$ is a class-accessible category strongly accessibly embedded in $\mathcal{K}$.

Proof.

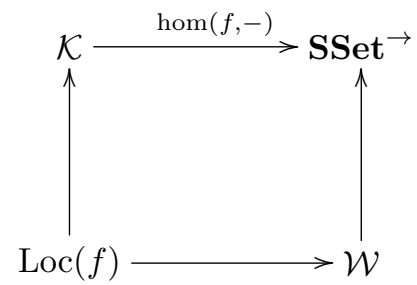

is a pullback where $\mathcal{W}$ denotes weak equivalences in SSet. Since the vertical leg on the right is transportable, $\operatorname{Loc}(f)$ is a pseudopullback and thus it is class-accessible and its inclusion to $\mathcal{K}$ is strongly class-accessible (see [11] 3.1, 3.2 and 2.12). Since

$$
\operatorname{Loc}(\mathcal{F})=\bigcap_{f \in \mathcal{F}} \operatorname{Loc}(f),
$$

the result follows from [11] 3.3.

Definition 3.4. Let $\mathcal{K}$ be a simplicial model category and $f: A \rightarrow B$ a cofibration of cofibrant objects. Consider a pushout

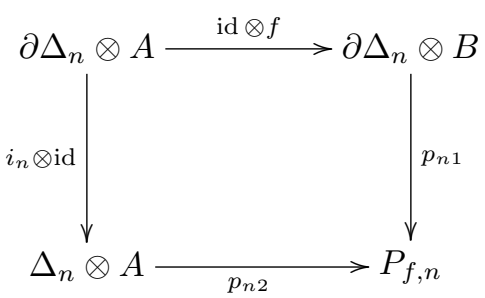

where $i_{n}: \partial \Delta_{n} \rightarrow \Delta_{n}$ is the inclusion of the boundary into a simplex. Let $h_{f, n}: P_{f, n} \rightarrow \Delta_{n} \otimes B$ be the canonical morphism, which is a cofibration since $\mathcal{K}$ is simplicial. 
Cofibrations $h_{f, n}, n=0,1, \ldots$ are called $f$-horns. If $\mathcal{F}$ is a class of cofibrations, then we denote by $\operatorname{Hor}(\mathcal{F})$ the collection of all $f$-horns, for all $f \in \mathcal{F}$.

Remark 3.5. Every $h_{f, n} \in \operatorname{Hor}(\mathcal{F})$ is an $\mathcal{F}$-local equivalence because the morphism

$$
\operatorname{hom}\left(h_{f, n}, K\right): \operatorname{hom}\left(\Delta_{n} \otimes B, K\right) \rightarrow \operatorname{hom}\left(P_{f, n}, K\right)
$$

is a weak equivalence for every $\mathcal{F}$-local object $K$. In fact, the morphism

$$
\operatorname{hom}(\mathrm{id} \otimes f, K): \operatorname{hom}\left(\Delta_{n} \otimes B, K\right) \rightarrow \operatorname{hom}\left(\Delta_{n} \otimes A, K\right)
$$

is a weak equivalence because $K$ is $\mathcal{F}$-local and $\operatorname{hom}\left(p_{n, 2}, K\right)$ is a trivial fibration as a pullback of the trivial fibration

$$
\operatorname{hom}(\mathrm{id} \otimes f, K): \operatorname{hom}\left(\partial \Delta_{n} \otimes B, K\right) \rightarrow \operatorname{hom}\left(\partial \Delta_{n} \otimes A, K\right) .
$$

Thus it suffices to use the 2-out-of-3 property.

We used the fact that $f$-horns are cofibrations between cofibrant objects and that the definition of an $\mathcal{F}$-local equivalence does not depend on the choice of a cofibrant approximation.

Lemma 3.6. Let $\mathcal{K}$ be a simplicial model category and $\mathcal{F}$ a class of cofibrations between cofibrant objects of $\mathcal{K}$. Then a fibrant object $K$ of $\mathcal{K}$ is $\mathcal{F}$-local if and only if it is injective to all $f$-horns for $f \in \mathcal{F}$.

Proof. Since each $f \in \mathcal{F}$ is a cofibration, $\operatorname{hom}(f, K)$ is a fibration for each fibrant object $K$. Thus a fibrant object $K$ is $\mathcal{F}$-local if and only if $\operatorname{hom}(f, K)$ is a trivial fibration for each $f \in \mathcal{F}$. This is the same as having the right lifting property with respect to each inclusion $i_{n}: \partial \Delta_{n} \rightarrow \Delta_{n}$. The latter is clearly equivalent to being injective with respect to $f$-horns $h_{f, n}$ for all $f \in \mathcal{F}$.

Lemma 3.7. Let $\mathcal{F}$ be a cone-coreflective class of cofibrations between $\lambda$-presentable cofibrant objects. Then $\operatorname{Hor}(\mathcal{F})$ is a cone-coreflective class of morphisms between $\lambda$ presentable objects.

Proof. Since $\partial \Delta_{n} \otimes B$ and $P_{f, n}$ are $\lambda$-presentable provided that $A$ and $B$ are $\lambda$ presentable, we have to prove that $\operatorname{Hor}(\mathcal{F})$ is cone-coreflective. Let $f: A \rightarrow B$ be an element of $\mathcal{F}$. Given a commutative square

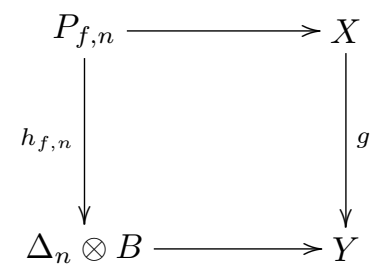


with $h_{f, n} \in \operatorname{Hor}(\mathcal{F})$ and $g$ arbitrary, we form, by adjunction, the following commutative square:

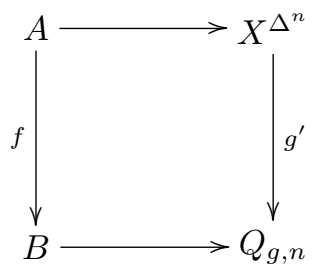

where $Q_{g, n}=X^{\partial \Delta^{n}} \times_{Y^{\partial \Delta^{n}}} Y^{\Delta^{n}}$.

Since $\mathcal{F}$ is cone-coreflective, there exists a set of morphisms $\mathcal{F}_{g^{\prime}}=\left\{f^{\prime}: A^{\prime} \rightarrow B^{\prime}\right\} \subset$ $\mathcal{F}$ such that any morphism $f \rightarrow g^{\prime}$ in $\mathcal{K} \rightarrow$ factors through some element $f^{\prime} \in \mathcal{F}_{g^{\prime}}$. Unrolling back the adjunction, we obtain the set of horns $\operatorname{Hor}\left(\mathcal{F}_{g^{\prime}}\right)=\left\{h_{f^{\prime}, n}: P_{f^{\prime}, n} \rightarrow\right.$ $\left.\Delta^{n} \otimes B^{\prime} \mid n \geqslant 0\right\}$ which depends entirely on $g$. Thus $\operatorname{Hor}(\mathcal{F})$ is cone-coreflective.

Remark 3.8. (1) Let $\mathcal{F}$ be a cone-coreflective class of cofibrations between $\lambda$-presentable cofibrant objects in a class $\lambda$-combinatorial simplicial model category $\mathcal{K}$. Then $\operatorname{Loc}(\mathcal{F})$ is weakly reflective and closed under $\lambda$-filtered colimits in $\mathcal{K}$ (following 3.7, 3.6 and [11] 4.4). Recall that a weak reflection $r_{K}: K \rightarrow K^{*}$ is obtained as a factorization

$$
K \stackrel{r_{K}}{\longrightarrow} K^{*} \longrightarrow 1 \text {. }
$$

in $\left(\operatorname{cof}(\operatorname{Hor}(\mathcal{F}) \cup \mathcal{C}),(\operatorname{Hor}(\mathcal{F}) \cup \mathcal{C})^{\square}\right)$, where $\mathcal{C}$ is a bounded class such that $\operatorname{cof}(\mathcal{C})$ are cofibrations in $\mathcal{K}$. Thus $r_{K}$ belongs to $\operatorname{cof}(\operatorname{Hor}(\mathcal{F}) \cup \mathcal{C})$. If $\mathcal{K}$ is left proper then, following $3.2(3), \operatorname{cof}(\operatorname{Hor}(\mathcal{F}) \cup \mathcal{C}) \subseteq \operatorname{cof}(\mathcal{C}) \cap \operatorname{LEq}(\mathcal{F})$. Hence $r_{K}$ is both a cofibration and an $\mathcal{F}$-local equivalence.

In order to apply the class version of Smith's theorem 2.15 we need to know that the local equivalences are class-accessible and strongly accessibly embedded. The only known technique to establish it is to represent the local equivalences as a class of maps taken into a class-accessible strongly accessibly embedded subcategory (usually a category of weak equivalences in a nice class-combinatorial model category) and then apply $[\mathbf{1 1}, 3.1]$.

Unfortunately we do not always know that the weak reflections constructed above are functorial. But in the case that there exists a functor $L: \mathcal{K} \rightarrow \operatorname{Loc}(\mathcal{F})$ and a natural transformation $\eta$ : Id $\rightarrow L$ such that $\eta_{K}$ is an $\mathcal{F}$-local equivalence for each $K$ in $\mathcal{K}$, such a functor $L$ is called an $\mathcal{F}$-localization functor.

(2) Given a model category $\mathcal{K}$ and a functor $L: \mathcal{K} \rightarrow \mathcal{K}$, then $\operatorname{LEq}(L)$ will denote the class of morphisms sent by $L$ to weak equivalences. If an $\mathcal{F}$-localization functor $L$ exists, then $\operatorname{LEq}(\mathcal{F})=\operatorname{LEq}(L)$.

In fact $h$ is an $\mathcal{F}$-local equivalence iff its cofibrant approximation $\tilde{h}$ is an $\mathcal{F}$-local equivalence. Since $\eta_{K}$ is an $\mathcal{F}$-local equivalence for each $K, \tilde{h}$ is an $\mathcal{F}$-local equivalence iff $L(\tilde{h})$ is an $\mathcal{F}$-local equivalence, i.e., a weak equivalence in $\mathcal{K}$ (see $3.2(2)$ ).

Proposition 3.9. Let $\mathcal{K}$ be a nice class-combinatorial model category and $L: \mathcal{K} \rightarrow$ $\mathcal{K}$ be a strongly class-accessible functor. Then $\mathrm{LEq}(L)$ is a class-accessible category strongly accessibly embedded in $\mathcal{K} \rightarrow$. 
Proof. By assumption, the class $\mathcal{W}$ of weak equivalences is class-accessible and strongly accessibly embedded in $\mathcal{K} \rightarrow$. Since $\operatorname{LEq}(L)$ is given by the pullback

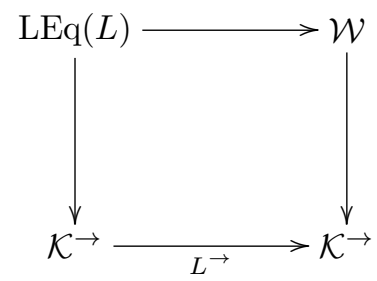

having the vertical leg on the right transportable, $\operatorname{LEq}(L)$ is a pseudopullback and thus class-accessible and strongly accessibly embedded in $\mathcal{K}^{\rightarrow}$ (see [11] 3.1 and 3.2).

Theorem 3.10. Let $\mathcal{K}$ be a nice, class-combinatorial, left proper, simplicial model category and let $\mathcal{F}$ be a class of morphisms in $\mathcal{K}$. Suppose there exists a strongly class-accessible $\mathcal{F}$-localization functor $L: \mathcal{K} \rightarrow \mathcal{K}$. Then the left Bousfield localization of $\mathcal{K}$ with respect to $\mathcal{F}$ exists if and only if the intersection of $L E q(\mathcal{F})$ with the cofibrations of $\mathcal{K}$ is a cone-coreflective class of morphisms.

Proof. Necessity immediately follows from the existence of the (trivial cofibration, fibration) factorizations in the localized model category cf. [11] $4.2(2)$ ).

In order to establish sufficiency, we will verify the conditions of 2.15. By 3.8 (2) and 3.9 the subcategory $\operatorname{LEq}(\mathcal{F})$ is class-accessible. There is a regular cardinal $\lambda$ such that $\operatorname{LEq}(\mathcal{F})$ is class- $\lambda$-accessible and $\mathcal{K}$ is class- $\lambda$-combinatorial. In fact, $\operatorname{LEq}(\mathcal{F})$ is class- $\mu$-accessible and $\mathcal{K}$ is class- $\nu$-combinatorial, it suffices to take $\mu, \nu \triangleleft \lambda$. Let $\mathcal{I}$ be the generating class of cofibrations in $\mathcal{K}$. Then $\mathcal{I}^{\square} \subseteq \operatorname{LEq}(\mathcal{F})$ because $\operatorname{LEq}(\mathcal{F})$ contains all weak equivalences. Following $3.2(3), \operatorname{cof} \mathcal{I} \cap \operatorname{LEq}(\mathcal{F})$ is closed under pushouts and transfinite compositions.

Example 3.11. Let $\mathcal{A}$ be a complete simplicial category. Then $\mathcal{P}(\mathcal{A})$ equipped with the projective model structure is a class-combinatorial model category (see 2.6). Let $f: V \rightarrow W$ be a cofibration of simplicial sets. Then the class $\mathcal{F}=\{f \otimes \operatorname{hom}(-, A) \mid$ $A \in \mathcal{A}\}$ is bounded. The argument is the same as in the proof of 2.6. The localization of $\mathcal{P}(\mathcal{A})$ with respect to $\mathcal{F}$ is equivalent to the levelwise localization with respect to $f$.

Let $L_{f}$ : SSet $\rightarrow$ SSet be the $f$-localization functor, i.e., a fibrant replacement functor in the $f$-localized model category structure on SSet. Then $L_{f}$ is finitely accessible provided that $V$ and $W$ are finitely presentable. Moreover, $L_{f}$ can always be chosen to be simplicial (see $[\mathbf{1 9}, 1.7]$ or $[\mathbf{2 9}, 24.2]$ ). Similarly to Lemma 2.8 , we get a strongly class-accessible simplicial functor $L: \mathcal{P}(\mathcal{A}) \rightarrow \mathcal{P}(\mathcal{A})$ assigning to $F$ the composition $L_{f} F$. Since $\operatorname{LEq}(L)=\operatorname{LEq}(\mathcal{F}), \operatorname{LEq}(\mathcal{F})$ is a class-accessible subcategory of $\mathcal{P}(\mathcal{A}) \rightarrow$ by 3.9 .

In a general case, $L_{f}$ is accessible and we would need an extension of 2.4 to $\lambda$-small weighted limits. This is valid but we have not burdened our paper with a proof.

Recall that if $\mathcal{K}$ is a model category, then a functor $L: \mathcal{K} \rightarrow \mathcal{K}$ equipped with natural transformation $\eta: \operatorname{Id}_{\mathcal{K}} \rightarrow L$ is called homotopy idempotent if $L \eta_{K}$ and $\eta_{L K}$ are weak equivalences for each $K$ in $\mathcal{K}$. 
Definition 3.12. Let $\mathcal{K}$ be a model category equipped with a homotopy idempotent functor $L: \mathcal{K} \rightarrow \mathcal{K}$ preserving weak equivalences. $L$-fibrations are the maps with the right lifting property with respect to cofibrations that are also $L$-equivalences. A left Bousfield localization of $\mathcal{K}$ with respect to $L$, or just an $L$-localization, is a new model structure on $\mathcal{K}$ such that the class of cofibrations coincides with the original class of cofibrations in $\mathcal{K}$ and the class of weak equivalences is $\operatorname{LEq}(L)$. New fibrations are the $L$-fibrations.

Theorem 3.13. Let $\mathcal{K}$ a nice, proper, simplicial class-combinatorial model category and $L: \mathcal{K} \rightarrow \mathcal{K}$ a strongly class-accessible homotopy idempotent functor preserving weak equivalences. Suppose additionally, that pullbacks of L-equivalences along $L$ fibrations are L-equivalences. Then the L-localization exists and is class-combinatorial.

Proof. It was shown in $[6$, Appendix A], that the pair

$$
\left(\operatorname{cof}(\mathcal{I}) \cap \operatorname{LEq}(L),(\operatorname{cof}(\mathcal{I}) \cap \operatorname{LEq}(L))^{\square}\right)
$$

is a weak factorization system. They argue as follows.

Take $i \in \operatorname{cof}(\mathcal{I}) \cap \operatorname{LEq}(L)$ and $f: X \rightarrow Y$. For any morphism $i \rightarrow f$ in $\mathcal{K} \rightarrow$ we perform the following construction:

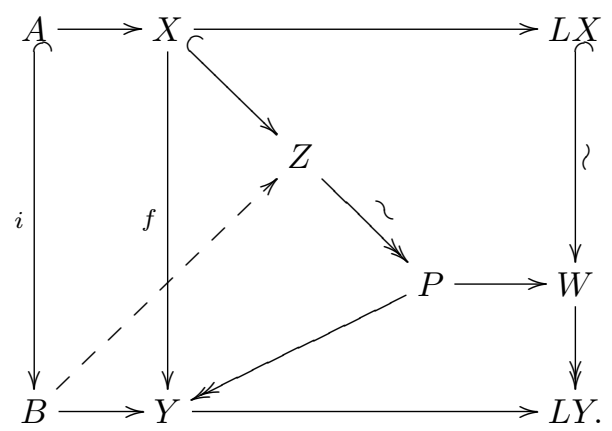

After applying the functor $L$ on the morphism $f$ we factor $L f$ as a trivial cofibration followed by a fibration in $\mathcal{K}$, obtaining the $L$-fibration $W \rightarrow L Y$, since this is a fibration of $L$-local objects [6, A.8(iii)]. Then constructing $P=W \times_{L Y} Y$ we obtain an $L$-fibration $P \rightarrow Y$ as a pullback of an $L$-fibration and an $L$-equivalence $P \rightarrow W$ due to the additional assumption. The induced morphism $X \rightarrow P$ is an $L$ equivalence by the 2-out-of-3 property. Now we factor the morphism $X \rightarrow P$ into a cofibration followed by a trivial fibration in $\mathcal{K}$. As the composition of two $L$-fibrations, the morphism $Z \rightarrow Y$ is an $L$-fibration, hence there exists a lift $B \rightarrow Z$, showing that $\operatorname{cof}(I) \cap \operatorname{LEq}(\mathcal{F})$ is cone-coreflective.

At this point we have already constructed the factorizations required for the localized model structure showing that the pair

$$
\left(\operatorname{cof}(\mathcal{I}) \cap \operatorname{LEq}(L),(\operatorname{cof}(\mathcal{I}) \cap \operatorname{LEq}(L))^{\square}\right)
$$

is a weak factorization system. But that was the contents of [6, Appendix A]. The novelty of our result is that this model structure is class-combinatorial again, hence it remains to show that this weak factorization system is cofibrantly class- $\lambda$-generated (see Definition 2.1). 
Like in the proof of 3.10 , there exists a regular cardinal $\lambda$ such that $\mathcal{K}$ is $\lambda$ combinatorial and $L$ strongly class- $\lambda$-accessible. Assume that $X$ and $Y$ are $\lambda$-presentable. Then $L X, L Y$ and $W$ are $\lambda$-presentable. By Lemma $2.4 P$ is also $\lambda$-presentable. Thus $Z$ is $\lambda$-presentable. Consequently, the $L$-localized model category is class- $\lambda$ combinatorial.

Example 3.14. Take $f: V \rightarrow 1$ in Example 3.11. For such maps $f$-localization functor is called also $V$-nullification. Then the resulting class of $f$-equivalences satisfies the conditions of 3.13, since the nullification of spaces (i.e., the localization with respect to $f$ for $f$ as above) is a right proper model category (see, e.g., [5]). Hence the model category resulting from the levelwise nullification of the projective model structure on the category of small functors is class-combinatorial again.

Example 3.15. Consider the category $\mathcal{P}\left(\mathbf{S S e t}^{\mathrm{op}}\right)$ of small simplicial functors from simplicial sets to simplicial sets equipped with the projective model structure (see 2.6). Consider the localization functor $L: \mathcal{P}\left(\right.$ SSet $\left.^{\mathrm{op}}\right) \rightarrow \mathcal{P}\left(\right.$ SSet $\left.^{\mathrm{op}}\right), \quad L=P_{n} \circ \mathrm{Fib}$, constructed in [3], where $P_{n}$ is Goodwillie's $n$-th polynomial approximation [17] and Fib: $\mathcal{P}\left(\right.$ SSet $\left.^{\mathrm{op}}\right) \rightarrow \mathcal{P}\left(\right.$ SSet $\left.^{\mathrm{op}}\right)$ is the strongly class-accessible fibrant replacement functor from 2.8. Since $P_{n}$ is a countable colimit of finite homotopy limits of cubical diagrams applied on homotopy pushouts (joins with finite sets used to construct $P_{n}$ in $[\mathbf{1 7}]$ may be expresses as homotopy pushouts), it is strongly class-accessible. Thus $L$ is strongly class-accessible, hence the polynomial model structure constructed in $[\mathbf{3}]$ is class-combinatorial.

The condition on the localization functor to be strongly class-accessible may not be omitted in 3.13 as the following example shows.

Example 3.16. The following localization of the class-combinatorial model category $\mathcal{P}($ SSet $)$ was constructed in $[\mathbf{9}]$. The localization functor $L: \mathcal{P}($ SSet $) \rightarrow \mathcal{P}($ SSet $)$ is the composition of the evaluation functor at the one point space ev $(F)=F(1)$ with

a fibrant replacement $\widehat{(-)}$ in simplicial sets (chosen to be a small functor like $\widehat{\operatorname{Id}_{\mathcal{S}}}$ or $\left.\mathrm{Ex}^{\infty}\right)$ and the Yoneda embedding $Y:$ SSet $\rightarrow \mathcal{P}($ SSet $)$, i.e., $L(F)=\operatorname{hom}(-, \widehat{F(1)})$. This localization functor satisfies the conditions of $A 6$ in [6] (pullback of an $L$ equivalence along an $L$-fibration is an $L$-equivalence again), and hence there exists the $L$-local model structure on $\mathcal{P}$ (SSet). The fibrant objects in the localized model category are levelwise fibrant functors weakly equivalent to representable functors, but they are not closed under filtered colimits, since filtered colimit of representable functors need not be representable, no matter how large the filtered colimit is. On the other hand, in a class-cofibrantly generated model category sufficiently large filtered colimits of fibrant objects are fibrant again. In other words, we obtain a localization of $\mathcal{P}$ (SSet), which is not class-cofibrantly generated. The reason is that the localization functor $L$ is not class-accessible. See [9] for more details on this model structure.

\section{Acknowledgements}

The first author acknowledges the hospitality of Masaryk University. The second author is supported by MSM 0021622409 and GAČR 201/11/0528. The hospitality of the Australian National University is gratefully acknowledged. 


\section{References}

[1] J. Adámek and J. Rosický, Locally Presentable and Accessible Categories, London Mathematical Society Lecture Note Series, vol. 189, Cambridge University Press, Cambridge, 1994.

[2] T. Beke, Sheafifiable homotopy model categories, Math. Proc. Cambr. Phil. Soc. 129 (2000), 447-475.

[3] G. Biedermann, B. Chorny and O. Röndigs, Calculus of functors and model categories, Advances in Mathematics 214 (2007), 92-115.

[4] F. Borceux, Handbook of Categorical Algebra 2. Categories and structures, Encyclopedia of Mathematics and its Applications, vol. 51, Cambridge University Press, Cambridge, 1994.

[5] A. K. Bousfield, On the telescopic homotopy theory of spaces, Trans. Amer. Math. Soc. 353 (2001), 2391-2426.

[6] A. K. Bousfield and E. M. Friedlander, Homotopy theory of $\Gamma$-spaces, spectra, and bisimplicial sets, Geometric Applications of Homotopy Theory, II, pp. 80130, Lect. Notes in Math.,vol. 658, Springer-Verlag, Berlin, 1978.

[7] B. Chorny, Localization with respect to a class of maps I. Equivariant localization of diagrams of spaces, Israel Journal of Math. 147 (2005), 99-140.

[8] B. Chorny, A generalization of Quillen's small object argument, J. Pure Appl. Alg. 204, (2006), no. 3, 568-583.

[9] B. Chorny, Brown representability for space-valued functors, Israel J. of Math., to appear.

[10] B. Chorny and W. G. Dwyer, Homotopy theory of small diagrams over large categories, Forum Math. 21 (2009), 167-179.

[11] B. Chorny and J. Rosický, Class-locally presentable and class-accessible categories, J. Pure Appl. Alg., to appear.

[12] J. D. Christensen and D. C. Isaksen, Duality and pro-spectra, Algebr. Geom. Topol. 4 (2004), 781-812.

[13] B. J. Day and S. Lack, Limits of small functors, J. Pure Appl. Alg. 210 (2007), 651-663.

[14] D. Dugger, Universal homotopy theories, Adv. Math. 164 (2001), 144-176.

[15] D. Dugger, Combinatorial model categories have presentations, Adv. Math. 164 (2001), 177-201.

[16] E. Dror Farjoun, Homotopy theories for diagrams of spaces, Proc. Amer. Math. Soc. 101 (1987), 181-189.

[17] T. G. Goodwillie, Calculus III: Taylor Series, Geom. $\& 3$ Top. 7 (2003), 645-711.

[18] P. G. Goerss and J. F. Jardine, Simplicial Homotopy theory, Progress in Mathematics, vol. 174, Birkhäuser Verlag, Basel, 1999.

[19] P. S. Hirschhorn, Model Categories and their Localizations, Mathematical Surveys and Monographs, vol. 99, American Mathematical Society, Providence, 2003. 
[20] M. Hovey, Model categories, Mathematical Surveys and Monographs, vol. 63, American Mathematical Society, Providence, 1999.

[21] D. C. Isaksen, A model structure on the category of pro-simplicial sets, Trans. Amer. Math. Soc. 353 (2001), 2805-2841.

[22] P. Karazeris, J. Rosický and J. Velebil, Completeness of cocompletions, J. Pure Appl. Alg. 196 (2005), 229-250.

[23] G. M. Kelly, Basic concepts of enriched category theory, London Mathematical Society Lecture Note Series, vol. 64, Cambridge University Press, Cambridge, 1982.

[24] G. M. Kelly, Structures defined by finite limits in the enriched context, I., Cahiers Top. Géom. Diff. 23 (1982), 3-42.

[25] J. Lurie, Higher Topos theory, Annals of Mathematics Studies, vol. 170, Princeton University Press, Princeton, 2009.

[26] M. Makkai and R. Paré, Accessible Categories: The Foundation of Categorical Model Theory, Cont. Math., vol. 104, American Mathematical Society, Providence, 1989.

[27] J. Rosický, Generalized Brown representability in homotopy categories, Theory Appl. Cat. 14 (2005), 451-479.

[28] J. Rosický, On combinatorial model categories, Appl. Cat. Str. 17 (2009), 303316.

[29] M. A. Shulman, Homotopy limits and colimits and enriched homotopy theory, arXiv:math.AT/0610194.

Boris Chorny chorny@math.haifa.ac.il

Department of Mathematics, University of Haifa at Oranim, Tivon, Israel

Jiří Rosický rosicky@math.muni.cz

Department of Mathematics and Statistics, Masaryk University, Faculty of Sciences Brno, Czech Republic 